'Departamento de Medicina Interna, Escuela de Medicina, Universidad de Valparaíso. Valparaíso, Chile.

${ }^{2}$ Centro de Investigaciones Biomédicas, Escuela de Medicina, Universidad de Valparaíso. Valparaíso, Chile.

Trabajo no recibió financiamiento. El autor declara no tener conflictos de interés.

Recibido el 11 de noviembre de 2019, aceptado el 18 de mayo de 2020.

Correspondencia:

Dr. Rienzi Díaz Navarro Universidad de Valparaíso, Calle Angamos 655, Viña del Mar, Chile. diaz.rienzi@gmail.com

\section{Transposición congénitamente corregida de grandes arterias en adultos asintomáticos diagnosticada circunstancialmente por ecocardiografía. Casos clínicos}

\author{
RIENZI DÍAZ-NAVARRO ${ }^{1,2}$
}

\section{Asymptomatic congenitally corrected transposition of the great arteries. Report of two cases}

\begin{abstract}
Congenitally corrected transposition of the great arteries is a rare congenital anomaly that can remain asymptomatic until adulthood, especially when there are no other associated congenital anomalies. We report two patients in their sixth decade of life with corrected transposition of the great arteries incidentally diagnosed by transthoracic echocardiography in a preventive medical check-up. The complementary use of cardiac computed tomography confirmed the diagnosis.

(Rev Med Chile 2020; 148: 868-874)

Key words: Echocardiography; Multidetector Computed Tomography; Transposition of Great Vessels.
\end{abstract}

\footnotetext{
L
} a transposición de las grandes arterias congénitamente corregida (TGACC), es una anomalía cardíaca congénita rara caracterizada por discordancia aurículo-ventricular y ventrículo-arterial representando menos de $1 \%$ de todas las cardiopatías congénitas ${ }^{1,2}$. La mayoría de los pacientes tienen otros defectos asociados, habitualmente comunicación interventricular, estenosis pulmonar y anormalidades de la válvula tricúspide ${ }^{3-6}$.

En la TGACC el ventrículo anatómicamente derecho (VD) funciona como ventrículo sistémico del que nace la aorta $(\mathrm{AO})$, mientras que el ventrículo anatómicamente izquierdo (VI) funciona como ventrículo pulmonar dando origen a la arteria pulmonar. La aurícula izquierda está conectada al VD a través de la válvula tricúspide y la aurícula derecha al VI a través de la válvula mitral. Así, la sangre venosa sistémica desoxigenada llega a la circulación pulmonar a través de la aurícula derecha, luego al VI a través de la mitral para alcanzar la arteria pulmonar. Por otra parte, la sangre venosa pulmonar oxigenada llega a la aurícula izquierda, luego al VD a través de la válvula tricúspide y finalmente a la AO. De esta manera, la circulación está fisiológicamente corregida y no hay cianosis (Figura 1).

Desde su descripción en $1875^{7}$, se han descrito numerosos casos de TGACC sin lesiones asociadas en pacientes adultos, algunos de los cuales han permanecido asintomáticos hasta la cuarta $o$ quinta década de vida, incluso hasta la octava ${ }^{8,9}$. Las complicaciones tardías de la TGA puede ser la insuficiencia tricuspídea, disfunción ventricular derecha, insuficiencia cardiaca derecha y bloqueo auriculoventricular completo ${ }^{8}$.

Comunicamos dos pacientes adultos con TGACC sin lesiones asociadas que fueron circunstancialmente pesquisados por ecocardiografía transtorácica y confirmados mediante tomografía cardíaca computada. 


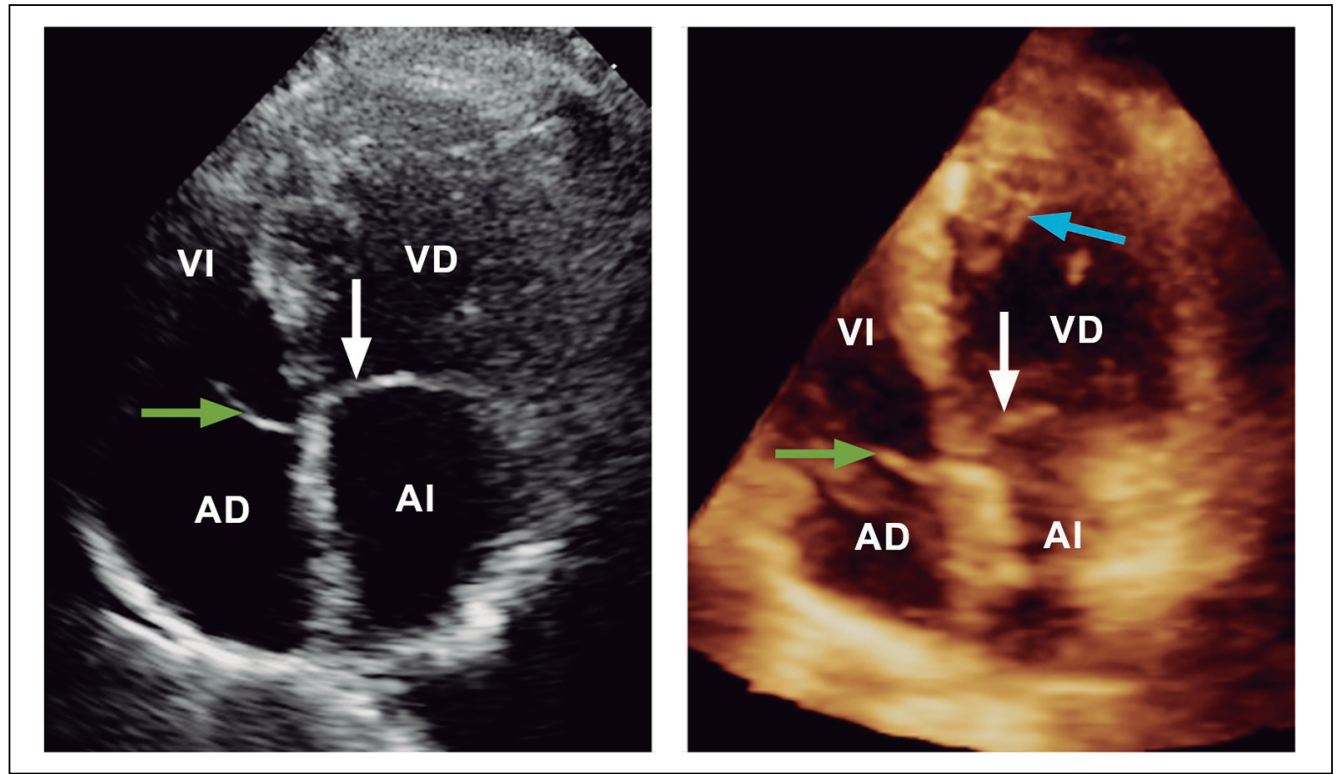

Figura 1. Ecocardiograma transtorácico 2D (izquierda) y 3D (derecha), visión de 4 cavidades. Se observa la válvula tricúspide con inserción en el septo interventricular desplazada hacia el ápex (Flechas blancas), respecto de la válvula mitral (Flechas verdes). La cavidad ventricular localizada a la izquierda tiene morfología de ventrículo derecho (VD) con abundante trabeculación y presencia de la banda moderadora (Flecha celeste). AD, aurícula derecha; Al, aurícula izquierda; VI, ventrículo izquierdo.

\section{Casos clínicos}

\section{Caso 1}

Mujer de 58 años, madre de dos hijos con embarazos de curso normal, asintomática y sin mórbidos. En control médico preventivo se realizó un electrocardiograma que evidenció onda T negativa en derivaciones D1 y AVL (Figura 1, panel superior). Se solicitó un ecocardiograma transtorácico para complementar estudio, identificándose la $\mathrm{AO}$ emergiendo de un ventrículo localizado a la izquierda con morfología de VD, conectado a la aurícula izquierda a través de la válvula tricúspide (Figura 1, panel inferior). Al mismo tiempo, se visualizó la arteria pulmonar emergiendo del VI localizado a la derecha. No se identificaron otras anomalías cardiacas y la función sistólica del VD era normal. En el examen cardiaco y pulmonar no se encontró anormalidades. Se realizó una tomografía cardíaca computada que confirmó el diagnóstico visualizándose la $\mathrm{AO}$ en posición anterior y a la izquierda de la arteria pulmonar (Figuras 2A y 2B) e identificándose el tronco coronario común izquierdo naciendo del seno aórtico anterior (Flechas blancas), para posteriormente dar origen a la arteria coronaria descendente anterior y la arteria circunfleja. Desde el seno aórtico posterior emerge la arteria coronaria que cursa a lo largo del surco aurículo-ventricular posterior entre el VD y la aurícula izquierda, luego de dar origen a un ramo marginal. Se confirma la discordancia atrio-ventricular con la aurícula izquierda conectada al VD y la aurícula derecha conectada al VI, así como discordancia ventrículo-arterial con la $\mathrm{AO}$ emergiendo desde el ventrículo derecho (Figura 2C, 2D, 2E) y la arteria pulmonar desde el VI (Figura 2F). No se identificó enfermedad coronaria ateroesclerótica.

\section{Caso 2}

Hombre de 59 años, asintomático, portador de hipertensión arterial en tratamiento con captopril. En control rutinario de su hipertensión se realizó un electrocardiograma que mostraba ondas $\mathrm{T}$ negativas en derivaciones D1 y AVL (Figura 3, panel superior) y un ecocardiograma transtorácico que identificaba la $\mathrm{AO}$ emergiendo de un ventrículo morfológicamente derecho, dilatado, hipertrófico y con disfunción sistólica leve con fracción de eyección de 47\% (Figura 3). No se 

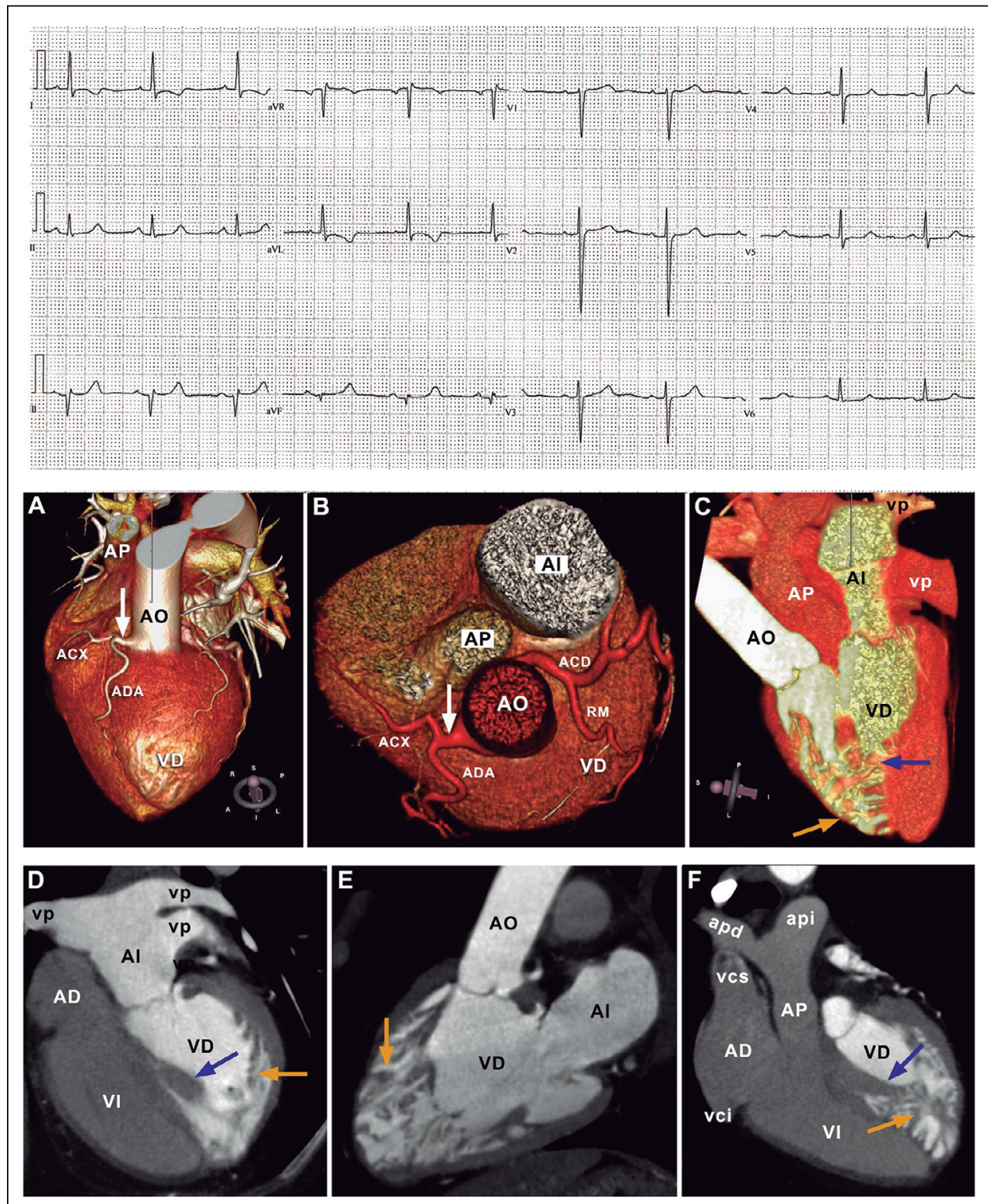

Figura 2. Panel superior: electrocardiograma con ritmo sinusal, intervalo PR normal, onda T negativa en D1-AVL y ausencia de onda Q en V5-V6. Panel medio: tomografía cardíaca computada con reformateo cardíaco tridimensional. A y B: relación espacial de las grandes arterias con la aorta $(A O)$ en posición anterior y a la izquierda de la arteria pulmonar (AP). Se observa, el tronco coronario izquierdo emergiendo desde el seno aórtico anterior (Flechas blancas) para luego dividirse en la arteria coronaria descendente anterior (ADA) y la arteria circunfleja ACX (Flechas blancas), y la arteria coronaria derecha (ACD) naciendo desde el seno aórtico posterior cursando a lo largo del surco aurículo-ventricular posterior entre el ventrículo derecho (VD) y la aurícula izquierda (Al), luego de dar origen a ramo marginal (RM) que irriga el VD. C: se identifica discordancia atrio-ventricular con la Al conectada al VD abundantemente trabeculado (Flecha naranja) y presencia de la banda moderadora (Flecha azul), además, discordancia ventrículo-arterial con la AO emergiendo desde el VD. Panel inferior: proyecciones de máxima intensidad. D y E: muestran la discordancia atrio-ventricular con la Al conectada al VD a través de la válvula mitral y la discordancia ventrículo-arterial con la AO emergiendo desde el VD abundantemente trabeculado (Flechas naranjas) y presencia de la banda moderadora (Flecha azul), ubicado a la izquierda del ventrículo izquierdo (VI). F: se observa el VI localizado a la derecha del VD conectado a la arteria pulmonar (AP). Abreviaciones: apd, arteria pulmonar derecha; api, arteria pulmonar izquierda; vci, vena cava inferior; vcs, vena cava superior; vp, vena pulmonar. 


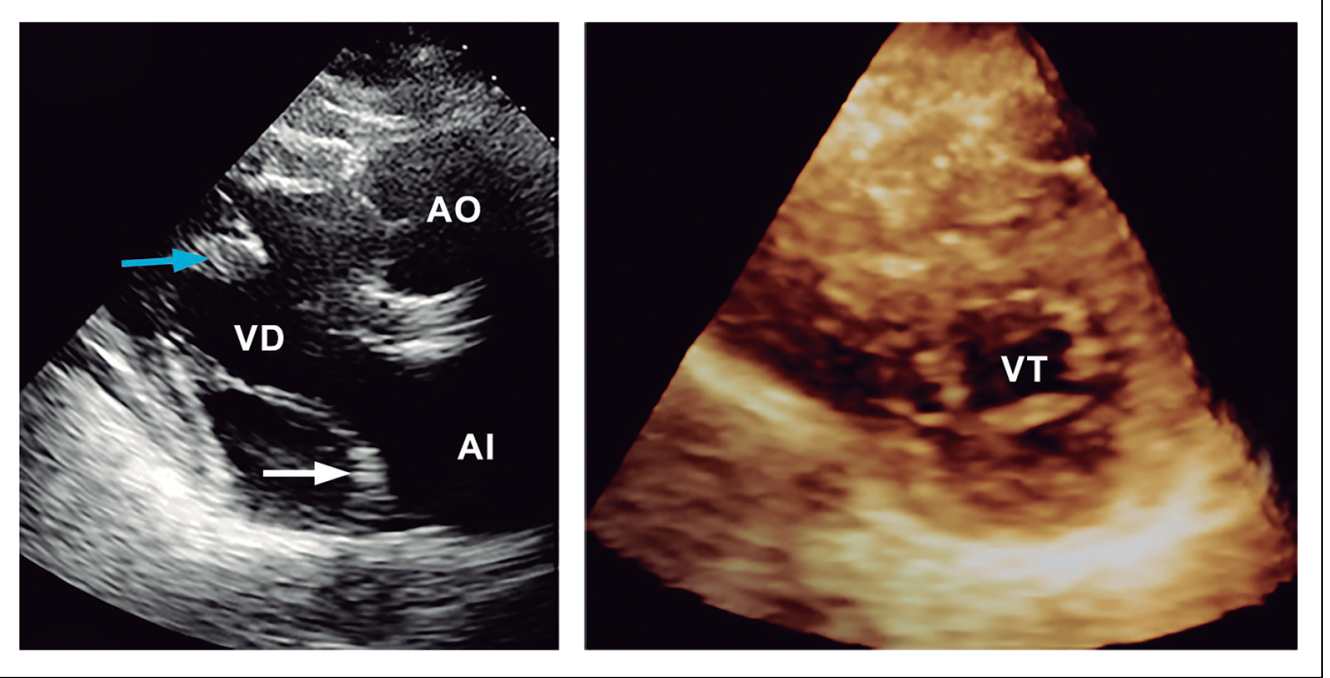

Figura 3. Izquierda: ecocardiograma transtorácico 2D, eje largo paraesternal izquierdo. Se observa, discordancia atrio-ventricular con la aurícula izquierda (AI) conectada al ventrículo derecho (VD) a través de la válvula tricúspide (Flecha blanca) y cono septal con la aorta $(\mathrm{AO})$ en posición anterior. La flecha celeste indica la banda moderada. Derecha: ecocardiograma transtorácico 3D, eje corto paraesternal izquierdo a nivel de la válvula tricúspide (VT) en diástole.

logró visualizar con claridad la emergencia de la arteria pulmonar y no se identificó otras anomalías cardiacas. El examen cardiaco y pulmonar fue normal. Con el diagnóstico de TGACC, se solicitó una tomografía cardíaca computada que confirmó el diagnóstico (Figura 4), identificándose el nacimiento del tronco coronario izquierdo desde el seno aórtico anterior (Figura 4A) y de la arteria coronaria derecha desde el seno aórtico posterior, reconociéndose también el origen por separado de un ramo marginal (Figura 2B). No se identificó ateromatosis coronaria.

\section{Discusión}

En la TGACC, existe discordancia aurículo-ventricular y ventrículo-arterial con las aurículas conectadas a sus ventrículos opuestos y los ventrículos a las arterias opuestas. Así, la sangre fluye desde la aurícula derecha al VI localizado al lado derecho del cuál emerge la arteria pulmonar y desde la aurícula izquierda al $\mathrm{VD}$ en posición izquierda desde donde emerge la $\mathrm{AO}^{3}$.

Existen dos tipos de TGACC, el situs solitus y el situs inverso. El situs solitus es el más frecuente con aurícula derecha a la derecha y la aurícula izquierda a la izquierda, ventrículos invertidos y levo-transposición de las grandes arterias con la $\mathrm{AO}$ en posición anterior y hacia la izquierda respecto de la arteria pulmonar. Los dos pacientes aquí comunicados son portadores de una TGACC tipo situs solitus. En 97\% de los casos de TGACC, hay anomalías congénitas asociadas ${ }^{10}$ y la mayoría de los pacientes desarrolla insuficiencia tricuspídea, disfunción del VD o bloqueo aurículoventricular de alto grado, complicaciones que ensombrecen el pronóstico ${ }^{1,3,11}$. Por otra parte, los pacientes sin lesiones asociadas pueden vivir asintomáticos hasta a la quinta década de su vida tal como nuestros pacientes ${ }^{8}$ y raramente son diagnosticados ${ }^{12}$. El electrocardiograma de los dos pacientes aquí comunicados, mostraba conducción aurículoventricular preservada con ausencia de onda $Q$ en derivaciones precordiales izquierdas que representarían despolarización septal inversa ${ }^{13}$.

En pacientes asintomáticos, el diagnóstico de TGACC se realiza habitualmente de manera circunstancial por ecocardiograma transtorácico, al identificarse el VD conectado con la aurícula izquierda y la AO localizada a la izquierda y en posición anterior de la arteria pulmonar. El VD se reconoce sin mayores dificultades por su abundante trabeculación y presencia de la banda modera- 


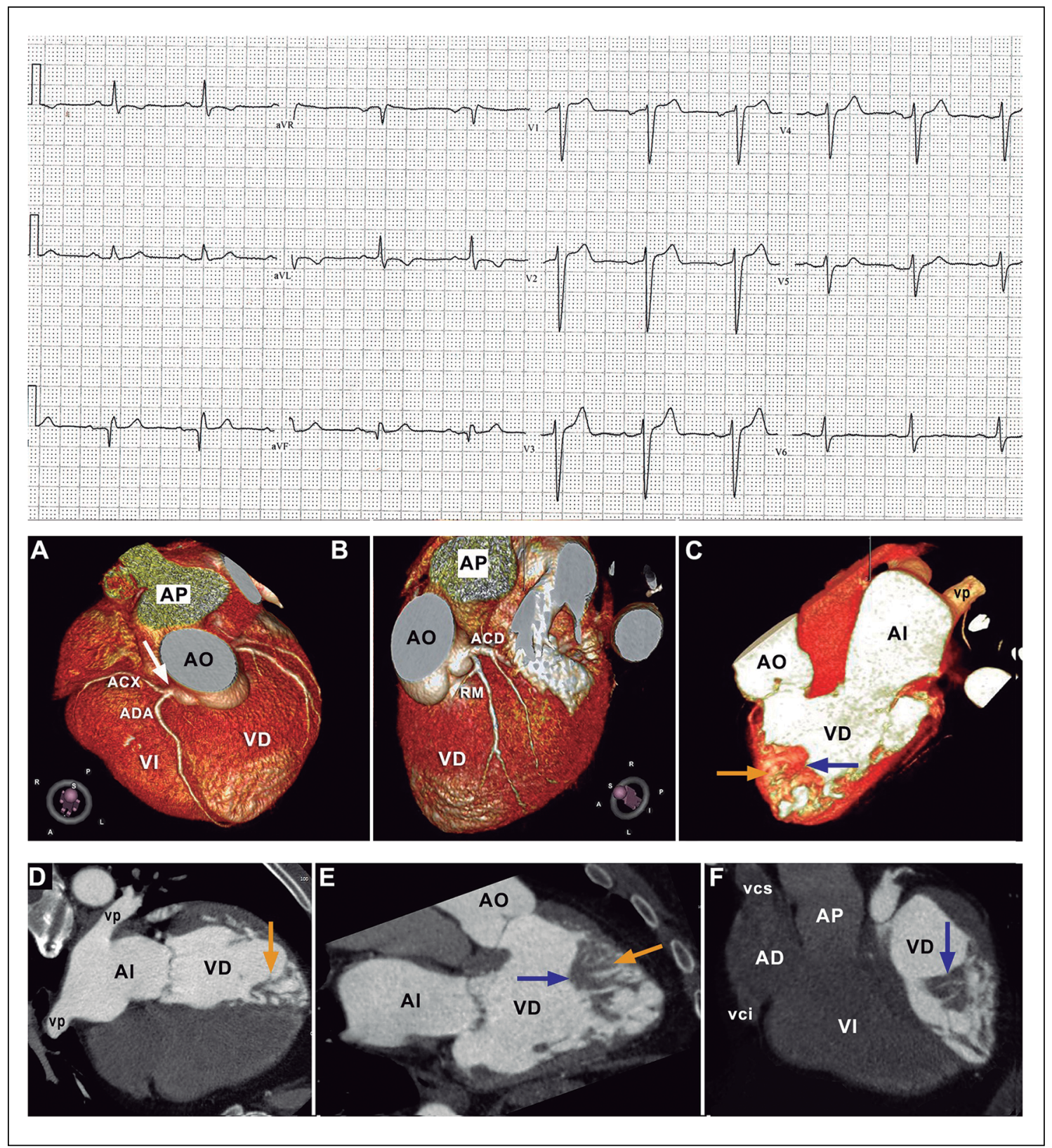

Figura 4. Panel superior: electrocardiograma con ritmo sinusal, intervalo PR de $200 \mathrm{~ms}$, onda T negativa en D1-AVL y ausencia de onda Q en precordiales izquierdas por despolarización septal inversa. Panel medio: tomografía computada de corazón con reformateo cardíaco tridimensional. A y B: Relación espacial de las grandes arterias con la AO en posición anterior y a la izquierda de la AP. Se observa el tronco coronario izquierdo emergiendo desde el seno aórtico anterior (Flecha blanca) que luego se divide en la arteria coronaria descendente anterior (ADA) y la arteria circunfleja ACX, y la arteria coronaria derecha (ACD) naciendo desde el seno aórtico posterior, además un ramo marginal (RM). C: discordancia atrio-ventricular con la aurícula izquierda (Al) conectada al ventrículo derecho (VD) abundantemente trabeculado (Flecha naranja) con la banda moderadora (Flecha azul) y discordancia ventriculoarterial con la aorta (AO) emergiendo desde el VD. Panel inferior: proyecciones de máxima intensidad. D y E: muestran discordancia atrio-ventricular con la Al conectada al VD, abundantemente trabeculado (Flechas naranjas) y con la banda moderadora (Flecha azul) y discordancia ventrículo-arterial con la AO emergiendo desde el VD. F: se observa el VI localizado a la derecha del VD dando origen a la arteria pulmonar (AP) y conectado a la AD y a la izquierda del VI, se reconoce el VD trabeculado con la banda moderadora (Flecha azul). 
dora, mientras que el VI de aspecto cónico y liso, se identifica conectado a la aurícula derecha y la arteria pulmonar, en posición anterior y a la derecha del VD. A su vez, la AO y la arteria pulmonar generalmente no se cruzan y emergen en paralelo desde los ventrículos opuestos. El diagnóstico de esta anomalía congénita se hace normalmente mediante ecocardiografía transtorácica, representa un desafío para el ecocardiografista pudiendo pasar inadvertida por las dificultades técnicas inherentes a este método diagnóstico e inexperiencia del operador $^{14}$. Por el contrario, la tomografía cardíaca computada permite diagnosticar o confirmar el diagnóstico con certeza, y pesquisar la existencia de otros defectos asociados, el origen y curso de las arterias coronarias y la presencia eventual de ateromatosis coronaria. En nuestros pacientes, el diagnóstico de TGACC fue hecho con ecocardiograma transtorácico y confirmado mediante tomografía cardíaca computada (Figuras 2 y 4 ).

La evolución de los pacientes con TGACC sin lesiones asociadas es mejor respecto de aquellos con más de un defecto congénito asociado ${ }^{1,3,5}$. Aproximadamente entre el $70-90 \%$ de estos pacientes, tienen malformaciones de la válvula tricúspide, no existentes en los casos aquí comunicados. La alteración anatómica de la válvula tricúspide representa un riesgo para el desarrollo de insuficiencia tricuspídea progresiva y es un factor independiente de mortalidad en la TGACC ${ }^{12}$. Sin embargo, la insuficiencia tricuspídea se puede desarrollar de igual manera producto de la carga de la presión sistémica ejercida sobre una válvula preparada para soportar menores cargas de presión, lo cual puede provocar disfunción del VD e insuficiencia cardiaca. Pero, es difícil afirmar con certeza si la insuficiencia tricuspídea produce disfunción de VD o viceversa ${ }^{5}$. Un mecanismo hipotético de disfunción VD podría ser la existencia de flujo coronario insuficiente para suplir el aumento de las necesidades de oxigenación durante el ejercicio, generando períodos de isquemia que causarían fibrosis y disfunción ventricular derecha progresiva e irreversible ${ }^{15}$. Ninguno de nuestros pacientes tenía insuficiencia tricuspídea, por lo tanto, la remodelación adversa del VD en el segundo caso (dilatación, hipertrofia y disfunción sistólica), es probablemente consecuencia de la elevada carga de presión sistémica sobre el VD, cuya morfología está nativamente condicionada para soportar cargas sistólicas de menor cuantía y fibrosis miocárdica secundaria a episodios de isquemia miocárdica intermitente ${ }^{13}$.

Los pacientes portadores de TGACC sin lesiones asociadas pueden vivir hasta la sexta década de la vida asintomáticos y su diagnóstico puede ser circunstancial. El ecocardiograma transtorácico es la técnica diagnóstica normalmente usada para su diagnóstico y la tomografía cardíaca computada es complementaria cuando la ecocardiografía presenta dificultades técnicas.

Ambos pacientes firmaron el consentimiento informado autorizando la publicación de sus casos clínicos anonimizados y respetando la confidencialidad.

\section{Referencias}

1. Connelly MS, Williams WG, Webb GD, Robertson P, McLauughlin PR. Congenitally corrected transposition of the great arteries in the adult: functional status and complications. J Am Coll Cardiol 1996; 27 (5): 1238-43.

2. Wallis GA, Debich-Spicer D, Anderson RH. Congenitally corrected transposition. Orphanet J Rare Dis 2011; 6: 22. doi: 10.1186/1750-1172-6-22.

3. Warnes CA. Transposition of the great arteries. Circulation 2006; 114 (24): 2699-709.

4. Ludstrom U, Bull C, Wyse RK, Somerville J. The natural and "unnatural" history of congenitally corrected transposition. Am J Cardiol 1990; 65 (18): 1222-9.

5. Graham TP Jr, Bernard YD, Mellen BG, Celermajer D, Baumgartner H, Cetta F, et al. Long-term outcome in congenitally corrected transposition of the great arteries: a multi-institutional study. J Am Coll Cardiol 2000;36 (1): 255-61.

6. Tay EL, Frogoudaki A, Inuzuka R, Giannahkpulas G, Prapa M, Li W, et al. Exercise intolerance in patients with congenitally corrected transposition of the great arteries relates to right ventricular filling pressures. Int J Cardiol 2011; 147 (2): 219-23.

7. Rokitanski C. Die Defecte Der Scheidewände Des Herzens: Pathologische Anatomische Abhandlung. Vienna, Austria: W. Braunmüller; 1875.

8. Presbitero P, Somerville J, Rabajoli F, Stone S, Conte MR. Corrected transposition of the great arteries without associated defects in adult patients: clinical profile and follow up. Br Heart J 1995; 74 (1): 57-9.

9. Placci A, Lovato L, Bonvivini M. BMJ Case Rep 2014. Doi: 10.1136/bcr-2014-204228.

10. Van Praagh R, Papagiannis J, Grünenfelder J, Bartram U, Martanovic P. Pathologic anatomy of corrected 
transposition of the great arteries: medical and surgical implications. Am Heart J 1998; 135 (5): 772-85.

11. Prieto LR, Hordof AJ, Secic M, Rosenbaum; Gersony WM. Progressive tricuspid valve disease in patients with congenitally corrected transposition of the great arteries. Circulation 1998; 98 (10): 997-1005.

12. Stout KK, Daniels CJ, Aboulhosn JA, Bozkurt B, Broberg CS, Colman JM, et al. 2018 AHA/ACC Guidelines for the Management of Adults With Congenital Herat Disease: A Report of the American College of Cardiology/ American Heart Asociation Task Force on Clinical Practice Guidelines. J AM Coll Cardiol 2019; 73 (12) e81-e192.
13. Sim MM. An electrocardigraphic telltate sign in a congenitally corrected transposition of the great arteries. J AM Coll Cardiol 2015; 65 (10S): A711.

14. Beauchesne LM, Warnes CA, Connolly HM, Ammash NM, Tajik AJ, Danielson GK. Outcome of the unoperated adult who presents with congenitally corrected transposition of the great arteries. J Am Coll Cardiol 2002; 40 (2): 285-90.

15. Hornung, TS, Bernard EJ, Celermajer DS, Jaeggi E, Howman-Giles RB, Chard RB, et al. Right Ventricular Dysfunction in Congenitally Corrected Transposition of the Great Arteries. Am J Cardiol 1990; 84 (9): 11169. 